\title{
EPAs, Milestones, Competences: Was brauchen wir davon?
}

\section{Sonia Frick}

KD Dr. med., MME, Leitende Ärztin, Innere Medizin / Intensivmedizin, Klinik für Innere Medizin, Spital Lachen (Schwyz)

Die grosse Trias von «knowledge, skills and attitudes» definiert heute alle Fähigkeiten einer Ärztin. Sie werden gelehrt, gelernt und auch geprüft. Doch wie sieht gutes Assessment aus? Wie baut man dies sinnvoll in ein Facharzt-Curriculum ein? Was braucht es dafür? Heisst die Antwort Competency-Based Medical Education (CBME)? Das engagierte Kader liest die aktuell überbordende Literatur zu diesem Thema und fühlt sich bald verloren. Dieser Artikel soll Ordnung in die Begrifflichkeiten bringen und die Begeisterung für diese Entwicklungen wecken.

\section{Competency-Based Medical Education}

CBME entstand in den sechziger Jahren als Kritik am «Tea-Bag Model» [1]: Bis dahin wurden Weiterzubildende wie ein Teebeutelchen für eine gewisse Zeit ins Wasser, also in die Weiterbildung "getaucht", ohne dass man gross dokumentierte, ob dabei auch eine «Wissensdiffusion» stattfand [2]. Das sollte sich ändern. Man wollte sich mehr auf den Inhalt statt der Dauer fokussieren. "It is an approach to preparing physicians for practice that is fundamentally oriented to graduate outcome abilities» [3]. Als Zweites sieht CBME vor, dass die Lernfortschritte kontinuierlich überprüft werden. Waren solche Überprüfungen während der Weiterbildung bis anhin eher als safety check gedacht
[4], reicht dies nicht mehr. Auf dieser Grundlage wurden die Inhalte und Instrumente von CBME entwickelt, die in der Folge vorgestellt werden.

\section{Kompetenzprofile}

Kompetenzprofile sind der grosse Überbau der CBME. Sie legen die professionellen Fähigkeiten (abilities) einer Medizinerin im ganzheitlichen Sinne von Wissen, Fertigkeiten und Haltung («knowledge, skills and attitudes») fest.

Kompetenzprofile sind im Vereinigten Königreich, in Holland, in den USA und in Kanada entwickelt worden. In der Schweiz haben wir uns für die verbreitetsten CanMEDs-Rollen entschieden. Unser allgemeiner Lernzielkatalog des SIWF baut darauf auf [5].

\section{Milestones}

Da die Kompetenzen doch ein theoretisches Konstrukt waren, war es nicht einfach, diese in der Praxis zu prüfen. Als Erstes entstanden dafür die Milestones, die vor allem in den USA Anwendung finden. Dazu wird der Lerninhalt einer jeden Kompetenz beschrieben und in vier bis fünf Weiterbildungsstufen unterteilt. Diese kurzen Beschriebe sind die Milestones: "A milestone will characterize expectations for residents at various stages of the development of expertise in a particular competency» [6]. Ein Beispiel:

- Beginn der Weiterbildung: Es können Informationen über einen Patienten gesammelt werden, so dass eine Diagnose gestellt werden kann. 
- Schluss der Weiterbildung: Es kann ein umfassendes Therapiekonzept erstellt werden.

\section{Entrustable Professional Activities (EPAs)}

Entrustable Professional Activities (EPAs) sind die jüngsten Evaluationsinstrumente der CBME. Statt vom Überbau der Kompetenzen auszugehen, prüfen EPAs eine Aufgabe oder eine Handlungseinheit aus dem medizinischen Alltag. Damit evaluiert man nicht nur eine, sondern verschiedene Kompetenzen, die in dieser Alltagssituation enthalten sind. Ein Beispiel: Um eine gute Visite durchzuführen, benötigt der Weiterzubildende die CanMEDs-Kompetenzen des Communicator, des Collaborator und des Medical Expert. Für jede EPA wird formuliert, wie die CanMEDs-Rollen darin sichtbar und prüfbar sind. Zum obigen Visitenbeispiel: Communicator: Drückt sich für den Patienten verständlich aus. Collaborator: Bezieht die Pflege in die Visite ein. Medical Expert: Verordnet das richtige Medikament.

Bei ihrer Entstehung waren EPAs detailliert beschrieben und vor allem für manuelle Fertigkeiten entwickelt worden (z.B. eine Blutentnahme durchführen); daraus ergaben sich für die ganze Weiterbildung sehr viele EPAs. Auch wurde jede Handlung einer EPA in einer Checkliste aufgeführt. Dies barg die Gefahr, dass es nur einen richtigen Ablauf, nämlich den auf der Checkliste, gab. Für komplexere Abläufe (obige Visite) war dies aber nicht praktikabel. Deshalb wurden die EPAs grösser und offener. Schlussendlich hat sich eine optimale Anzahl von 20-30 EPAs für ein ganzes Curriculum von 4-6 Jahren ergeben.

Für die EPAs existieren fünf Levels, auf welchen sie ausgeführt werden können (Tab. 1). Eine EPA wird von den Weiterzubildenden also mit wachsender Selbständigkeit ausgeführt, bis sie im Level 4 als «entrustable», also anvertraubar, gilt [9]. Die einzelnen EPAs bleiben also durch die ganze Weiterbildungszeit meist die gleichen, und die Weiterzubildenden arbeiten an den verschiedenen EPAs parallel.

EPAs werden geprüft, indem verschiedene Beobachter die Weiterzubildenden zu mehreren Zeitpunkten in mehreren Situationen beurteilen. Dies kann durchaus nur eine kurze Sequenz aus einer EPA sein (Beispiel

Tabelle 1: Skalierung für EPAs (AMEE Guide No. 99) [8].

\begin{tabular}{ll}
\hline Level 1 & Beobachtet \\
\hline Level 2 & Arbeitet unter direkter proaktiver Supervision (Supervisor im Raum) \\
\hline Level 3 & Arbeitet unter indirekter Supervision (Supervisor jederzeit abrufbar) \\
\hline Level 4 & Arbeitet ohne Supervision (Supervisor vorhanden, aber nicht vor Ort) \\
\hline Level 5 & Kann jüngere Kollegen supervidieren \\
\hline
\end{tabular}

Visite: entweder die Patientenkommunikation oder das Verordnen etc). Dies geschieht durch Arbeitsplatzbasierte Assessments (AbAs).

\section{Arbeitsplatzbasierte Assessments (AbAs)}

In den AbAs (englisch: Workplace-based Assessments, WBA) findet alles statt: das Assessment, das Feedback, der Lernprozess. In Tabelle 2 sind einige AssessmentMethoden zu einer Toolbox zusammengestellt.

Die entscheidende Frage ist nun, wann eine Aktivität den Level 4 erreicht hat, also als «entrustable» gilt. Eine Antwort liegt in der Beurteilungsbreite: Je mehr Beurteilungen von verschiedenen Weiterbildnern für eine EPA vorliegen, desto besser kann diese beurteilt werden; je komplexer die EPA, desto mehr AbAs braucht es dazu. Durch die Wahl verschiedener AbAs können auch verschiedene Teilbereiche (nested EPAs) innerhalb einer EPA getestet werden. In der Zusammenschau aller durchgeführten ABAs kann somit entschieden werden, ob die Weiterzubildende in dieser EPA «entrustable» ist.

\section{Lernzielkataloge}

Lernzielkataloge sind die ältesten Dokumente in der Weiterbildung, bilden sie doch das "knowledge» innerhalb der Trias «knowledge, skills and attitudes» ab. Sie haben aber sehr an Bedeutung verloren. In den kompetenzbasierten Curricula dienen sie am ehesten als Rahmen für die schriftlichen Prüfungen.

\section{E-Portfolio}

Ohne ein digitales Instrument, in welchem die Assessments aufgezeichnet werden können, können CBMEbasierte Curricula nicht implementiert werden. Für die Schweiz wäre ein E-Portfolio anstelle des bestehenden e-Logbuches sinnvoll. Die aktuelle Revision soll als Chance genutzt werden.

\section{Vor- und Nachteile der CBME-Instrumente}

Die Entwicklung der CBME war von jeher umstritten [2]. Kleinkrämerisch, detailorientiert und ressourcenverschleissend sei sie - und versuche die medizinische Kunst durch die Kompetenzen in Einzelteile zu zerlegen, die dem Berufsbild nicht gerecht würden [10]. Die Kompetenzen seien zu theoretisch, die Meilensteine zu praxisfern, die EPAs zu aufwendig. Trotzdem haben sich diese Instrumente durchgesetzt. Am besten hat sich das Zusammenspiel von Kompetenzen und EPAs bewährt. 
Tabelle 2: Auswahl Arbeitsplatzbasierter Assessments (AbAs).

\begin{tabular}{|c|c|}
\hline Workplace-based Assessments & Erklärungen \\
\hline Reflective Practice & Schriftliche Reflexion über eine klinische Situation und die eigene Rolle darin \\
\hline Case-based Discussion & Leiten oder Beteiligung bei fallbasierten Diskussionen \\
\hline Chart reviews & $\begin{array}{l}\text { Durchsicht der KG-Führung: Sinnhaftigkeit der Einträge, Vollständigkeit } \\
\text { der Diagnoseliste, Austrittsberichte }\end{array}$ \\
\hline Ward rounds & Beurteilung einer vollständigen Visite \\
\hline Professional presentations & Beurteilung in Präsentationen (Kongressen etc.) \\
\hline Journal Clubs & Beurteilung der wissenschaftlichen Aufarbeitung eines Artikels \\
\hline Publications & Qualität von Publikationen \\
\hline $\begin{array}{l}\text { Objective structured clinical examinations } \\
\text { (OSCE) }\end{array}$ & $\begin{array}{l}\text { Beurteilung einer fallbasierten Situation mit Schauspielpatienten, } \\
\text { zum Beispiel eines Herzinfarkts }\end{array}$ \\
\hline Multi-Source Feedback (MSF) & $\begin{array}{l}\text { Meist } 360-\text {-Grad-Feedback, inklusive Patienten und paramedizinischen } \\
\text { Personals, zum Beispiel Physiotherapie }\end{array}$ \\
\hline Patient Satisfaction & Feedback von Patienten in Form von direkten Feedbacks oder Fragebogen \\
\hline
\end{tabular}

In den meisten Ländern ist es das Kader, welches die EPAs durchführt, und die Leiter der Weiterbildungsstätten sind für die regelmässige Beurteilung der Portfolios zuständig. Die Investition in deren Schulung darf nicht unterschätzt werden (faculty development). In den Niederlanden heisst dies mindestens eine Woche Kader-Training pro Jahr.

In den Ländern, wo zentrale Institutionen die Reports der Weiterzubildenden ebenfalls erhalten, zum Beispiel zur Beurteilung der Weiterbildungsstätten (USA, England), werden zusätzliche Fragen aufgeworfen: Wie viele und welche Daten dürfen öffentlich gemacht werden? Wem gehört das E-Portfolio? Ein Gerichtsfall in England zeigt die Wichtigkeit dieser Diskussion [11].

\section{Praktisches für die Schweiz}

Die kontinuierliche Evaluation als einer der wichtigsten Bausteine für Qualität und Professionalität kommt in unserem aktuellen System zu kurz. Für Veränderungen bestehen aber gute Voraussetzungen: In der universitären Ausbildung werden aktuell die «PROFILES» eingeführt (Principal Relevant Objetives and a Framework for Integrative Learning and Education in Switzerland) [12]. Dieses Dokument beinhaltet die Lernziele des Studiums. Ab 2020 wird sich die Eidgenössische Schlussprüfung des Medizinstudiums daran orientieren. Die Profiles enthalten sowohl die CanMEDs-Rollen als auch EPAs. Künftig werden also Ärzte auf den Arbeitsmarkt drängen, die mit diesen Frameworks ver- traut sind. Deshalb haben sie bei einer guten und rationalen Anwendung auch für die Weiterbildung das grösste Potential. Für die Weiterzubildenden sind sie ein Kontrollwerkzeug in der eigenen Weiterbildung (Assessment for Learning). Für die Supervisoren sollen sie Leitlinien für die Überprüfung der Weiterzubildenden sowie auch der eigenen Lehrstätte sein (Assessment of Learning).

\section{Die Vorteile}

In der Schweiz fehlen longitudinale, stark strukturierte Residency Programs wie in anderen erwähnten Ländern. Unsere Programme sind dezentral und modular. Das macht die kontinuierliche Evaluation und Förderung schwieriger. Bei einer durchdachten Anwendung können die EPAs jedoch eine gute Lösung für dieses Problem bieten: Sie decken durch ihre Levels die ganze Entwicklung in den verschiedenen Weiterbildungsjahren ab und schaffen so eine logische Verbindung zwischen den einzelnen Stellen.

Für die Weiterbildungsstättenleiter heisst dies, dass sie nach einer Analyse ihrer Weiterbildungsstätte definieren können, welche Lernziele und welche EPAs sie abdecken. Das schärft das Profil der Weiterbildungsstätten und macht diese im besten Fall attraktiver. Eventuell würde dies auch den Zusammenschluss von einzelnen Weiterbildungsstätten zur Bildung eines kompletten Weiterbildungscurriculums anstossen, wie es aktuell in Bern für die Allgemeine Innere Medi- 
zin entsteht [13] und anderorts auch für die Chirurgie bereits besteht.

Ein weiterer Punkt: Wenn bei einem Stellenwechsel anhand der EPAs der Wissensstand für die nächste Stelle einheitlich dargestellt sichtbar ist und sogar die Basis für die neuen Lernziele bildet, wird die Weiterbildung stringenter, der Einsatz der Weiterzubildenden in den Kliniken sinnvoller, und Ressourcen werden so besser genutzt.

\section{Ausblick}

Die Weiterbildung zu allen Facharzttiteln würde von einer klugen Umsetzung von CBME sehr profitieren. Idealerweise können - als grosse Vision - auch fächerübergreifende EPAs geschaffen werden (Visiten, Vorstellung eines Patienten, Reanimationen etc.) und so anstelle des allgemeinen Lernzielkatalogs eine praxisorientierte Verbindung zwischen allen Fachrichtungen geschaffen werden.

Der Zeitpunkt für eine Entwicklung dieser Instrumente ist richtig. Das zeigt auch das Interesse an der Konstituierung einer interdisziplinären Arbeitsgruppe «Entrustable professional activities» des SIWF im Juli 2018. Will man EPAs erstellen, existieren dazu bereits Guidelines [8] und einfach anwendbare Tools [14]. Einige Fachgesellschaften wie die Anästhesie haben damit bereits begonnen.

Natürlich muss man sich der Grösse des Projekts bewusst sein. Betroffen sind alle: Leiter und Kader der Weiterbildungsstätten müssen in ihrer Funktion als Weiterbildungsverantwortliche gestärkt werden. Dazu werden sie Zeit und Engagement einsetzen müssen, sowohl in die eigene Schulung als Supervisoren als auch in die alltägliche Applikation und Dokumentation der Instrumente der CBME. Die Weiterzubildenden müssen sich vermehrt selbst um ihr E-Portfolio bemühen. Unsere übergeordneten Gremien wie das SIWF sind gefordert, weil die höheren Anforderungen für die Anerkennung eines Facharzttitels konsequent implementiert werden müssen. Politischer Wille ist nötig, der diese Entwicklung auch fördert und den Instrumenten eine gewisse Verbindlichkeit und Wichtigkeit gibt. Dies alles bedingt also insofern ein Umdenken, als Lehre und Evaluation noch einmal deutlich an Wichtigkeit gewinnen. Die Rollen des Coachs, Supervisors und Assessors werden noch expliziter zu einem zentra- len Teil unseres Berufs. Das Schlusswort gehört einer holländischen Expertin: «It all depends on the quality, and honesty - and often courage - of the assessors. Without that, whatever system you chose, it will always fail.» Es ist uns zu wünschen, dass wir diese Herausforderung annehmen.

\section{Disclosure statement}

Diese Arbeit ist aus meiner Masterthese für den MAS in Medical Education an der Universität Bern entstanden. Der Originaltitel lautet «Milestones, Entrustable Professional Activities, Portfolios: Was brauchen wir? Assessments im internationalen Vergleich und die Implikationen für das Weiterbildungscurriculum in Allgemeiner Innerer Medizin der Schweiz». Die Arbeit wurde im Sommer 2018 angenommen und der Titel des MAS in Medical Education im Herbst 2018 verliehen. Sie wurde bisher nirgends publiziert.

Die Autorin arbeitet an verschiedenen Universitätetn (Zürich, Fribourg, Lugano).

\section{Literatur}

1 O. ten Cate, Competency-Based Postgraduate Medical Education Past, Present and Future, German Medical Science GMS Publishing House, Nov. 2017.

2 L. S. Snell and J. R. Frank, Competencies, the tea bag model, and the end of time, Med. Teach., vol. 32, no. 8, pp. 629-30, Aug. 2010.

3 J. R. Frank, R. Mungroo, Y. Ahmad, M. Wang, S. De Rossi, and T. Horsley, Toward a definition of competency-based education in medicine: a systematic review of published definitions, Med. Teach., vol. 32, pp. 631-7, Jul. 2010

4 L. D. Gruppen, O. ten Cate, L. A. Lingard, P. W. Teunissen, and J. R. Kogan, Enhanced Requirements for Assessment in a CompetencyBased, Time-Variable Medical Education System, Acad. Med., vol. 93, pp. S17-S21, Mar. 2018.

5 B. Bloom, Taxonomy of educational objectives: the classification of educationaal goals.

6 D. P. Sklar, Competencies, Milestones, and Entrustable Professional Activities: What They Are, What They Could Be, Acad. Med., vol. 90 no. 4, pp. 395-7, Apr. 2015.

7 D. R. Taylor, et al. Creating Entrustable Professional Activities to Assess Internal Medicine Residents in Training: A Mixed-Methods Approach, Ann. Intern. Med. 168(10):724-9 (2018)

8 O. ten Cate, H. C. Chen, R. G. Hoff, H. Peters, H. Bok, and M. van der Schaaf, Curriculum development for the workplace using Entrustable Professional Activities (EPAs): AMEE Guide No. 99, Med. Teach., vol. 37, no. 11, pp. 983-1002, 2015.

9 O. ten Cate, Nuts and bolts of entrustable professional activities, J. Grad. Med. Educ., vol. 5, no. 1, pp. 157-8, Mar. 2013.

10 C. Carraccio, S. D. Wolfsthal, R. Englander, K. Ferentz, and C. Martin, Shifting paradigms: from Flexner to competencies, Acad. Med. J. Assoc. Am. Med. Coll., vol. 77, no. 5, pp. 361-7, May 2002.

11 Hadiza Bawa-Garba case, Wikipedia. 28-Apr-2018.

12 L. S. Verte, PROFILES | Home [online], available: http://profilesmed. ch/ [accessed: 04-May-2018].

13 S. Streit, M. Perrig, N. Rotondi, and D. Aujesky, Das Berner Curriculum für Allgemeine Innere Medizin, Schweiz Ärzteztg., vol. 99(21), May 2018

14 D. R. Taylor, et al., EQual, a Novel Rubric to Evaluate Entrustable Professional Activities for Quality and Structure, Acad. Med. J. Assoc. Am. Med. Coll., vol. 92, no. 11S; Association of American Medical Colleges Learn Serve Lead: Proceedings of the 56th Annual Research in Medical Education Sessions, pp. S110-S117, 2017. 